\title{
Monitoring Method of Cow Anthrax Based on Gis and Spatial Statistical Analysis
}

\author{
Lin $\mathrm{Li}^{1}$, Yong Yang ${ }^{1,3, *}$, Hongbin Wang ${ }^{2}$, Jing Dong ${ }^{1}$, Yujun $\mathrm{Zhao}^{1}$, Jianbin $\mathrm{He}^{1}$, \\ and Honggang Fan $^{2}$
}

${ }^{1}$ Faculty of Animal Husbandry and Veterinary Medicine, Shenyang Agricultural University, 110161 Shenyang, P.R. China

lilin619619@yahoo.com.cn

${ }^{2}$ Faculty of anima Medicine 1, Northeast Agricultural University, 150030 Harbin, P.R. China

${ }^{3}$ Faculty of Information and Electric Engineering, Shenyang Agricultural University,

110161 Shenyang, P.R. China

yangsyaul163. com

\begin{abstract}
Geographic information system (GIS) is a computer application system, which possesses the ability of manipulating spatial information and has been used in many fields related with the spatial information management. Many methods and models have been established for analyzing animal diseases distribution models and temporal-spatial transmission models. Great benefits have been gained from the application of GIS in animal disease epidemiology. GIS is now a very important tool in animal disease epidemiological research. Spatial analysis function of GIS can be widened and strengthened by using spatial statistical analysis, allowing for the deeper exploration, analysis, manipulation and interpretation of spatial pattern and spatial correlation of the animal disease. In this paper, we analyzed the cow anthrax spatial distribution characteristics in the target district A (due to the secret of epidemic data we call it district A) based on the established GIS of the cow anthrax in this district in combination of spatial statistical analysis and GIS. The Cow anthrax is biogeochemical disease, and its geographical distribution is related closely to the environmental factors of habitats and has some spatial characteristics, and therefore the correct analysis of the spatial distribution of anthrax cow for monitoring and the prevention and control of anthrax has a very important role. However, the application of classic statistical methods in some areas is very difficult because of the pastoral nomadic context. The high mobility of livestock and the lack of enough suitable sampling for the some of the difficulties in monitoring currently make it nearly impossible to apply rigorous random sampling methods. It is thus necessary to develop an alternative sampling method, which could overcome the lack of sampling and meet the requirements for randomness. The GIS computer application software ArcGIS9.1 was used to overcome the lack of data of sampling sites.Using ArcGIS 9.1 and GEODA to analyze the cow anthrax spatial distribution of district A. we gained some conclusions about cow anthrax' density: (1) there is a spatial clustering model. (2) there is an intensely spatial autocorrelation. We established a prediction model to estimate the anthrax distribution based on the spatial characteristic of the density of cow
\end{abstract}

${ }^{*}$ Corresponding author.

D. Li and C. Zhao (Eds.): CCTA 2009, IFIP AICT 317, pp. 21-26, 2010.

(c) IFIP International Federation for Information Processing 2010 
anthrax. Comparing with the true distribution, the prediction model has a well coincidence and is feasible to the application. The method using a GIS tool facilitates can be implemented significantly in the cow anthrax monitoring and investigation, and the space statistics - related prediction model provides a fundamental use for other study on space-related animal diseases.

Keywords: GIS, spatial statistic analysis, animal epidemiology, cow anthrax.

\section{Introduction}

Geographic information system (GIS) is powerful automated system for the capture, storage, retrieval, analysis, and display of spatial data. The system offers new and expanding opportunities for epidemiology because it allows an informed user to choose between options when geographic distributions are part of the problem. Even when used minimally, this system allows a spatial perspective on disease. Used to their optimum level, as tools for analysis and decision making, they are indeed a new information management vehicle with a rich potential for animal epidemiological research. Epidemiologists have traditionally used maps when analyzing associations between location, environment, and the disease (Busgeeth, K. et al., 2004). GIS is particularly well suited for studying these associations because of its spatial analysis and display capabilities. Recently GIS has been used in the surveillance and monitoring of vector-borne diseases (Glass GE, et al., 1995; Beck LR, et al., 1994; Richards FO, et al.,1993; Clarke KC, et al., 1991), water borne diseases (Braddock M, et al.,1994), in environmental health (Barnes S and Peck A. 1994; Wartenberg D, et al., 1993; Wartenberg D. et al.,1992), and the analysis of disease policy and planning (Marilyn O Ruiz, et al.,2004).Spatial analysis function of GIS can be widened and strengthened by using spatial statistical analysis, allowing for the deeper exploration, analysis, manipulation and interpretation of spatial pattern and spatial correlation of the animal disease.

Anthrax is a disease of warm-blooded animals, including humans, most livestock and some wildlife. It is caused by the spore-forming bacteria Bacillus anthracis. Herbivorous animals are highly susceptible to anthrax, For cow, the disease usually is acute, resulting in death in one to three days. By the time cow displays signs of disease, including staggering, trembling, convulsions, or bleeding from body openings, death usually follows quickly. Cow anthrax is biogeochemical disease and primarily occurs in alkaline soils with high nitrogen levels caused by decaying vegetation, alternating periods of rain and drought. Its geographical distribution is related closely to the environmental factors of habitats and has some spatial characteristics, and therefore the correct analysis of the spatial distribution of anthrax cow for monitoring and the prevention and control of anthrax has a very important role.

However, the application of classic statistical methods in some areas is very difficult because of the pastoral nomadic context. The high mobility of livestock and the lack of enough suitable sampling for the some of the difficulties in monitoring currently make it nearly impossible to apply rigorous random sampling methods. It is thus necessary to develop an alternative sampling method, which could overcome the lack of sampling and meet the requirements for randomness. 


\section{Materials and Methods}

In this paper, we analyzed the cow anthrax spatial distribution characteristics in the target district A (due to the secret of epidemic data we call it district A) based on the established GIS of the cow anthrax in this district in combination of spatial statistical analysis and GIS. We established a prediction model to estimate the anthrax distribution based on the spatial characteristic of the density of cow anthrax. A twostage random sampling was used. It consisted in generating sampling sites at random and then drawing the required number of cow from the nearest herd to each point, and this, for practical reasons, within a specified radius. In this sampling method, the primary unit was defined as a settlement, watering point or grazing area where animals were expected to be found. Due to a lack of an exhaustive list of these locations in some areas and because of the high mobility of pastoral herds, the unpredictability of their movement, the classical random selection of sites was not feasible. Therefore, there was the need to develop an alternative method that allows random selection of the first sampling units. To that effect, GIS computer application software (ArcGIS) was used.

\subsection{Cow Anthrax Data Sampling Based on ArcGIS}

ArcGIS Spatial analyst is an extension to ArcGIS Desktop that provides powerful tools for comprehensive, raster-based spatial modeling and analysis. Using ArcGIS Spatial Analyst, you can derive new information from your existing data, analyze spatial relationships, build spatial models, and perform complex raster operations. With its extension to generate random coordinates, ArcGIS is able to generate at random the required number of sites within the area where sampling needs to take place, be it at zone, country, region or even district level, simultaneously allowing the application of weight factors to different sub-units of the surveyed area. Each site is identified by its longitude and latitude coordinates. In reality, a generated site is materialized only as a point on the map and therefore a fixed radius is defined around each point in order to determine a geographical area within which the sampling of cow anthrax is carried out. The determination of length of the radius takes into consideration the cow anthrax density. A radius below $10 \mathrm{~km}$ in a dry area may result in too many sampling sites with no animals, whereas in a densely populated area around permanent watering points and grazing areas, a relatively shorter radius suffices. A surplus of the total number of sites is generated at random to replace target sites that could not be accessed due to natural obstacles or sites with no animals within the defined circle.

\subsection{Cow Anthrax Density Spatial Cluster Analysis}

Spatial cluster analysis plays an important role in quantifying geographic variation patterns. It is commonly used in disease surveillance, spatial epidemiology, population genetics, landscape ecology, crime analysis and many other fields, but the underlying principles are the same. Spatial clustering analysis is the main research field of spatial data mining. We analysis cow anthrax data spatial cluster using High/Low Clustering tool in ArcGIS which can measures the degree of clustering for either high 
values or low values. The tool calculates the value of $\mathrm{Z}$ score for a given input feature class. A z-score is a measure of the divergence of an individual experimental result from the most probable result, the mean. $\mathrm{Z}$ is expressed in terms of the number of standard deviations from the mean value.

$$
z=\frac{x-\mu}{\sigma}
$$

where: $\mathrm{x}$ is ExperimentalValue, $\mu$ is Mean, and $\sigma$ is StandardDeviation.

This $\mathrm{z}$-value or $\mathrm{z}$ score expresses the divergence of the experimental result $\mathrm{x}$ from the most probable result $\mu$ as a number of standard deviations $\sigma$ The larger the value of $\mathrm{z}$, the less probable the experimental result is due to chance. For this tool, the null hypothesis states that the values associated with features are randomly distributed. The higher (or lower) the $\mathrm{Z}$ score, the stronger the intensity of the clustering. A Z score near zero indicates no apparent clustering within the study area. A positive $Z$ score indicates clustering of high values. A negative $\mathrm{Z}$ score indicates clustering of low values.

\subsection{Cow Anthrax Density Spatial Autocorrelation Analysis}

Spatial Autocorrelation is correlation of a variable with itself through space. If there is any systematic pattern in the spatial distribution of a variable, it is said to be spatially autocorrelation. If nearby or neighboring areas are more alike, this is positive spatial autocorrelation, Negative autocorrelation describes patterns in which neighboring areas are unlike. Random patterns exhibit no spatial autocorrelation. Spatial autocorrelation is important, because most statistics are based on the assumption that the values of observations in each sample are independent of one another. Positive spatial autocorrelation may violate this, if the samples were taken from nearby areas .Goals of spatial autocorrelation Measure is the strength of spatial autocorrelation in a map and test the assumption of independence or randomness. Spatial autocorrelation is, conceptually as well as empirically, the two-dimensional equivalent of redundancy. It measures the extent to which the occurrence of an event in an area unit constrains, or makes more probable, the occurrence of an event in a neighboring area unit. We analysis cow anthrax data Spatial Autocorrelation using Global Moran's I tool in GEODA. Moran's I is a measure of spatial autocorrelation developed by Patrick A.P. Moran. Moran's I is defined as:

$$
I=\frac{N}{\sum_{i} \sum_{j} w_{i j}} \frac{\sum_{i} \sum_{j} w_{i j}\left(X_{i}-\bar{X}\right)\left(X_{j}-\bar{X}\right)}{\sum_{i}\left(X_{i}-\bar{X}\right)^{2}}
$$

where: $\mathrm{N}$ is the number of spatial units indexed by $\mathrm{i}$ and $\mathrm{j}, \mathrm{x}$ is the variable of interest,

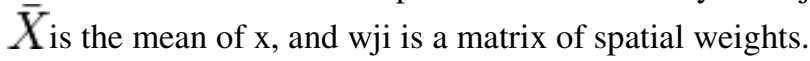

Negative (positive) values indicate negative (positive) spatial autocorrelation. Values range from -1 (indicating perfect dispersion) to +1 (perfect correlation). A zero values indicate a random spatial pattern. For statistical hypothesis testing, Moran's I values can be transformed to Z-scores in which values greater than 1.96 or smaller than -1.96 indicate spatial autocorrelation that is significant at the $5 \%$ level. 


\section{Results and Discussion}

The results of cow anthrax density spatial cluster analysis showed that the density of dairy cattle anthrax distribution in space with a high concentration of the Z Score = 5.6, $\mathrm{P}<0.01$, which means that there is a spatial clustering model. The results of Cow anthrax density spatial Autocorrelation analysis showed Moran's I $=0.4358$, which means there is an intensely spatial autocorrelation. Based on the spatial characteristic of the density of cow anthrax we established a prediction model to estimate the anthrax distribution by Ordinary Kriging tool in ArcGIS. Ordinary kriging (OK) is a geostatistical approach to modeling. Instead of weighting nearby data points by some power of their inverted distance, OK relies on the spatial correlation structure of the data to determine the weighting values. Ordinary Kriging is a spatial estimation method where the error variance is minimized. Superimposing map of predict map (Fig1) and distribution of cow anthrax epidemic focus in district A (Fig2). Comparing with the true distribution, the prediction model has a well coincidence and is feasible to the application.

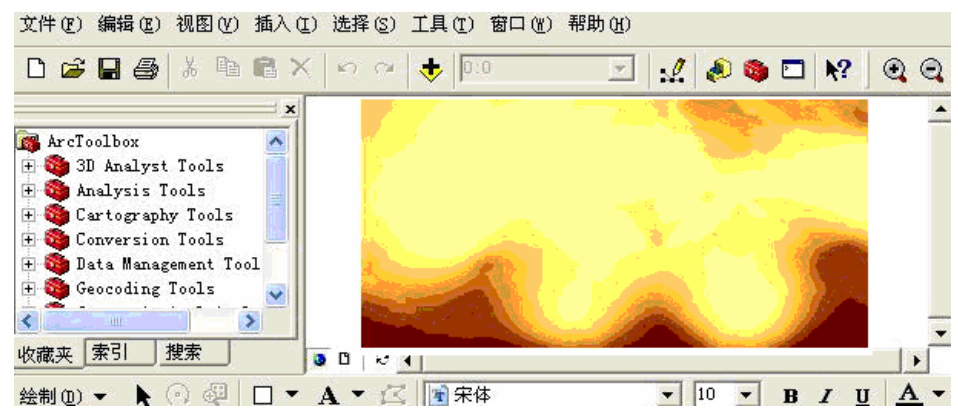

Fig. 1. Predict map of cow anthrax neural distribution in district A

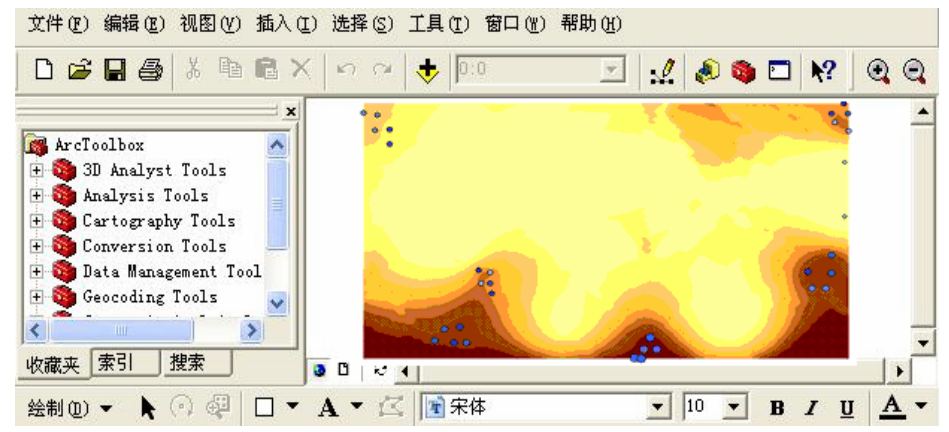

Fig. 2. Superimposing map of predict and the true distribution of cow anthrax neural 


\section{Conclusion}

GIS are powerful computerized systems with capabilities for inputting, storage, mapping, analysis and display of spatial data associated with a location on the Earth's surface. GIS have tremendously enhanced ecological epizootiology, the study of diseases in relation to their ecosystems. They have found increasing application for surveillance and monitoring studies, identification and location of environmental risk factors as well as disease prediction, disease policy planning, prevention and control. In this article we discuss the application of GIS to veterinary and medical research and the monitoring method of cow anthrax based on GIS and spatial statistical analysis. The method using a GIS tool facilitates is implemented significantly in the cow anthrax monitoring and investigation, and the space statistics - related prediction model provides a fundamental use for other study on space-related animal diseases.

\section{Acknowledgements}

Funding for this research was in part provided by the postdoctoral fund of Shenyang Agricultural University, P. R. China. The authors are grateful to the Shenyang Agricultural University for providing conditions with finishing this research.

\section{References}

Busgeeth, K., et al.: The use of a spatial information system in the management of HIV/AIDS in South Africa. International Journal of Health Geographics 3, 13 (2004)

Glass, G.E., Schwartz, B.S., Morgan III, J.M., Johnson, D.T., Noy, P.M., Israel, E.: Environmental risk factors for Lyme disease identified with geographic information systems. Am. J. Public Health 85, 944-948 (1995)

Beck, L.R., Rodrigues, M.H., Dister, S.W., Rodrigues, A.D., Rejmankova, E., Ulloa, A., et al.: Remote sensing as a landscape epidemiologic tool to identify villages at high risk for malaria transmission. Am. J. Trop. Med. Hyg. 51, 271-280 (1994)

Richards Jr., F.O.: Use of geographic information systems in control programs for onchocerciasis in Guatemala. Bull. Pan. Am. Health Organ. 27, 52-55 (1993)

Clarke, K.C., Osleeb, J.R., Sherry, J.M., Meert, J.P., Larsson, R.W.: The use of remote sensing and geographic information systems in UNICEF's dracunculiasis (Guinea worm) eradication effort. Prev. Vet. Med. 11, 229-235 (1991)

Braddock, M., Lapidus, G., Cromley, E., Cromley, R., Burke, G., Branco, L.: Using a geographic information system to understand child pedestrian injury. Am. J. Public Health 84, 1158-1161 (1994)

Barnes, S., Peck, A.: Mapping the future of health care: GIS applications in Health care analysis. Geographic Information systems 4, 31-33 (1994)

Wartenberg, D., Greenberg, M., Lathrop, R.: Identification and characterization of populations living near high-voltage transmission lines: a pilot study. Environ. Health Perspect 101, 626-632 (1993)

Wartenberg, D.: Screening for lead exposure using a geographic information system. Environ. Res. Dec. 59, 310-317 (1992)

Ruiz, M.O., Tedesco, C., McTighe, T.J.: Connie Austin and Uriel Kitron. International Journal of Health Geographics 3, 8 (2004) 